(C) 1984. The Genetical Society of Great Britain.

\title{
SELF INCOMPATIBILITY IN LOLIUM: A REPLY
}

\author{
W. SPOOR AND J. M. MCCRAW \\ The Edinburgh School of Agriculture, West Mains Road, Edinburgh EH9 3JG
}

Received 1.xii.83

In a recent issue of this journal (Heredity, 51,2), a paper was published criticising the work and approach adopted by us in the examination of self-incompatibility in Lolium spp. (Heredity, 50, 1, 21-27, 29-33). We would like to take this opportunity afforded by the Editor to reply to the criticism expressed by Lawrence and co-workers.

Three areas of criticism are identified:

1. Failure to recognise classes of pollination

In exploratory surveys, both at Edinburgh and Reading, pollen compatibility counts were carried out. The results obtained showed a continuous variation in the range of percentage compatible pollen among different crosses which could not be described as representing any distinct classes of pollen compatibility (a similar effect was found by B. G. Murray, working with Briza spicata, p.c.). Thus in our material we were unable to assign individual crosses as 50 per cent, 75 per cent and 100 per cent pollen compatible and did not feel that visual assessment would be sufficiently accurate. In addition, crosses showing less than 50 per cent pollen compatibility were observed, a phenomenon not expected under the two locus system.

In the published work of Lawrence and co-workers, no figures are available of counts of compatible/incompatible pollen grains, and hence no standard errors. In a single cross one would expect the values to differ from the expected by chance and therefore one would expect to see some statistical justification for recognising only four levels of compatibility. Indeed one would be interested to see if there was any overlapping between classes, 50-75 per cent, 75-100 per cent. Certainly this would require a great deal of work. Because of the above difficulties, we decided to recognise only two categories, compatible and incompatible, Families were investigated fully to highlight the genetic control of self-incompatibility in Lolium spp.

2. Absence of a model

The use of a model when investigating a genetic problem has many benefits when the experimental techniques are proven to be sufficiently sensitive to reveal real differences. Although Lawrence and co-workers are sure that they can distinguish between four different classes of pollen compatibility we were not, and hence decided that to choose a model would be inappropriate. Under this heading, Lawrence and co-workers also discuss the dangers of misclassifying pollinations and point out that in our work we have indicated when self pollen has successfully produced a few pollen tubes although this was reflected in zero or very low percentage seed set. Lawrence $e t$ al. then argue that these self-compatible pollinations should be regarded as self-incompatible and state that we have made a serious misclassification. They then relate this type of "misclassification" to other 
crosses in each diallel. This assumption by Lawrence and co-workers is erroneous. The aim of the study was to investigate self-incompatibility, a phenomenon which, in grasses, normally takes place at the stigmatic surface. The fact that with certain plants we were repeatedly observing self pollen growth through the stigma, however slight, was real, and therefore ought to be reported. To suggest that these observations are misclassifications which may be reflected in the whole body of work is misleading.

3. Interpretation of data

Lawrence $e$ t al. show that the probability of obtaining families with each member unique with respect to incompatibility reaction cannot be explained unless one concludes that five or more genes are involved in the reaction behaviour. While this may be true the results we have presented are an accurate record of the observations made during the study. Certainly we agree that systems of such complexity would be difficult to elucidate completely with only one round of pollinations. However, within the time available to us, it was possible to complete only one round. Nevertheless, we felt that the results we obtained were worthy of publication in that they indicated a complex incompatibility system.

We hope that the above points have clarified the reasons for the methods used in the investigation of the genetic control of self-compatibility in Lolium spp., and the interpretation of the results obtained.

\section{Information In Blological Systems}

The Role of Macromolecules

\section{WERNER HOLZMULLER \\ Translated by MANFRED HECKER}

This comprehensive account of information theory is written for students of all branches of natural science. It puts forward the argument that man cannot be the result of some mechanistic coincidence, but that there must be a plan underlying the evolution of life which extends Darwin's theory of the survival of the fittest and which is reflected in modern ecology. About 17.50 net

\section{The Neutral Theory of Molecular Evolution MOOTO KIMURA}

'a work of great significance, which should be read by everyone with a serious interest in evolution.' New Scientist

'This is the book we have been waiting for ...A milestone in evolution biology' John Maynard Smith Nature £35.00 net

\section{CAMBRIDGE UNIVERSITY PRESS}

